\title{
Surgical Procedures Used for Correction of Scheuermann's Kyphosis: A Meta-Analysis
}

\author{
Qingshan Li $\mathbb{C}$ \\ Fourth Department of Orthopedics, Handan Central Hospital of Hebei Province, Handan 056001, China \\ Correspondence should be addressed to Qingshan Li; 751105518@qq.com
}

Received 15 September 2021; Accepted 9 October 2021; Published 23 October 2021

Academic Editor: Sidong Yang

Copyright (C) 2021 Qingshan Li. This is an open access article distributed under the Creative Commons Attribution License, which permits unrestricted use, distribution, and reproduction in any medium, provided the original work is properly cited.

\begin{abstract}
Objectives. Scheuermann's kyphosis can cause severe back pain and cosmetic disorders to patients. Previous studies on surgical procedure selection for correction of Scheuermann's kyphosis have drawn controversial conclusions. Here, a meta-analysis was performed to figure out a better way between anterior-posterior (AP) combined procedures and posterior-only (PO) procedures. Methods. We searched PubMed database and Ovid database, as well as Cochrane Library (between January 2009 and December 2020, around recent ten years), for studies reporting Scheuermann's kyphosis correction in an anterior way or a posterior way. Random effects meta-analysis regarding correction degrees and incidence of proximal junctional kyphosis (PJK) was performed. Results. Finally, 13 unique studies including 586 patients (AP: 300; PO: 286) were identified and included for this meta-analysis. Overall, 6 AP cohorts and $10 \mathrm{PO}$ cohorts were pooled regarding the correction degrees of kyphosis in the analysis, respectively. Pooled correction degrees in AP cohorts were 33.31 (95\% CI: 27.48-39.15; $I^{2}=86 \%, P<0.001$ ) and in PO cohorts were 31.16 (95\% CI: 26.97-35.35; $\left.I^{2}=81.1 \%, P<0.001\right)$. Comparison of correction between AP and PO cohorts did not indicate any significant difference. Likewise, postoperative PJK incidence showed no difference. Back pain can be caused by both AP and PO procedures, but which causes less pain remains to be conclusive. The PO approach showed less blood loss and shorter surgical duration as compared to the AP approach. Conclusions. In summary, this meta-analysis shows similar treatment effects between AP and PO procedures in correcting Scheuermann's kyphosis, suggesting the advantage of PO procedures due to less blood loss and surgical duration. However, the postoperative complications PJK and distal junctional kyphosis (DJK) cannot be well concluded due to the limitation of existing data.
\end{abstract}

\section{Introduction}

Scheuermann's kyphosis (SK) is a rigid developmental thoracic kyphosis, which can cause severe back pain and cosmetic disorders to patients [1,2]. Although conservative treatment measures are initially applied, surgical treatment is indicated for kyphosis that is over 70-75 degrees, with significant pain that has not responded to conservative management, and/or respiratory problems due to severe kyphosis, and neurological issues [3-5]. The surgical treatment consists of two different ways; one is the combined anterior-posterior approach (AP) and the other is a posterior-only way $(\mathrm{PO})$, with various types of anchors $[6,7]$.

However, previous studies on surgical procedure selection for correction of SK have drawn contradictory conclusions. It was reported that a sufficient correction can be achieved by the PO approach, but the AP approach was more likely to get into a satisfying correction [8]. By contrast, a comparative study reported that the PO approach was more successful with a lower incidence of complications, as compared to the AP approach [9]. Interestingly, Koller et al. [10] found that both approaches achieved similar degrees of correction with higher fusion level in the PO group, after comparing the AP with $\mathrm{PO}$ procedures in correcting kyphosis.

Considering the controversy stated above, in this study, a meta-analysis was performed in order to figure out a better way between $\mathrm{AP}$ and $\mathrm{PO}$ procedures in treating SK patients.

\section{Materials and Methods}

2.1. Ethical Statement. The ethical approval was waived because all analyses were based on previously published studies. 
2.2. Literature Search. We searched PubMed database and Ovid database, as well as Cochrane Library (between January 2009 and December 2020, around recent ten years), for studies reporting SK correction in an anterior way or a posterior way. Articles should be written and published in English. Literature search for studies of interest should include the following terms: (1) Scheuermann's kyphosis AND posterior fusion or (2) Scheuermann's kyphosis AND anterior fusion.

2.3. Inclusion Criteria. All included studies should have at least reported the outcome of kyphosis correction, recruiting a cohort of Scheuermann's disease patients who underwent AP surgery or PO surgery, regardless of comparative or noncomparative studies. Here, we focus on studies of surgical procedure selection for correction of SK based on the effect of kyphosis correction.

2.4. Data Extraction. First, all related article titles and abstracts were screened and only original research was included. Second, full-length relevant articles were intensively read and checked in detail. At last, baseline information was extracted, as well as the raw data regarding follow-up time, patient age, sex distribution, sample size, Cobb angle, correction degrees, correction rate, blood loss, surgical duration, and postoperative complications including proximal junctional kyphosis (PJK) and distal junctional kyphosis (DJK).

2.5. Quality Assessment of Included Studies. All included studies in this meta-analysis were retrospective case-control studies or observational cohort studies. Thus, Newcastle-Ottawa quality assessment scale ( 9 points) was suitable for quality assessment and used to evaluate the quality of included studies [11].

2.6. Measures of Treatment Effect. Both continuous and dichotomous outcomes were generated in this study. Weighted mean difference (WMD) and 95\% confidence interval (CI) were generated for continuous outcomes. Also, the odds ratio (OR) and 95\% CI were calculated for dichotomous outcomes.

2.7. Assessment of Heterogeneity. Distributed as $\chi^{2}$ statistics, $Q$ statistics was used to evaluate heterogeneity, with its $P$ values revealed by the forest plot. The heterogeneity test was considered statistically significant when $P<0.10$. Simultaneously, $I^{2}$ was used to estimate the size of the heterogeneity. $I^{2}>50 \%$ indicated considerable heterogeneity among the included studies, and then a random effects analysis should be performed in meta-analysis.

2.8. Test for Risk of Publication Bias. Funnel plot was not performed to determine risk of publication bias due to the small number of included studies. Begg's and Egger's tests were used to assess the publication bias.
2.9. Statistical Analysis. All data analyses were conducted with software STATA 12.0 (Stata Corporation, College Station, TX, USA). Random effects meta-analysis regarding correction degrees and incidence of PJK was performed. Heterogeneity was assessed by $I^{2}$ statistic. $P$ values were set at 0.10 as significant in assessment of heterogeneity, Begg's test, and Egger's test $[12,13]$. In the rest of all, $P<0.05$ was regarded as statistically significant. All $P$ values were presented as two-tailed.

\section{Results}

3.1. Literature Search. As presented in Figure 1, after database search, there were 95 relevant papers included in the first-round literature selection. After study selection, 13 unique studies $[4,6,10,14-22]$ including 586 patients (AP: 300; PO: 286) were identified and included for this metaanalysis. Overall, 6 AP cohorts and 10 PO cohorts were pooled regarding the correction degrees of kyphosis in the analysis, respectively. Three reports were excluded due to unavailability of raw data [23-25].

3.2. Quality Assessment of Included Studies. A summary of quality assessment for each included study is shown in Table 1. Overall, three studies scored 7 points, eight scored 8 points, and two scored 9 points. The methodological quality of all included studies was found to be relatively high.

3.3. Characteristics of Included Studies. As shown in Tables 2-4, we extracted baseline information and relevant raw data regarding follow-up time, patient age, sex distribution, sample size, Cobb angle, correction degrees, correction rate, blood loss, surgical duration, and postoperative complications including PJK and DJK. All studies were retrospective in design. Follow-up time ranged from 22.8 months to 216 months. Patient age was between 11 and $44 \pm 8$ years. Also, most patients were males.

3.4. Pooled Analysis of Kyphosis Correction. As shown in Figure 2, six studies $[6,10,16,19,21,22]$ reported the correction effect by AP and were pooled into the metaanalysis. As a result, pooled correction degrees in AP cohorts were 33.31 (95\% CI: 27.48-39.15; $I^{2}=86 \%, P<0.001$ ). Because the study (Koller et al. [10]) might have recruited in the AP cohort 46 patients that were included in another study (Koller et al. [19]), we have revised the pooled analysis of AP group with the study (Koller et al. [10]) excluded; then the pooled correction degrees in AP cohorts were 33.45 (95\% CI: 25.97-40.92; $\left.I^{2}=88.8 \%, P<0.001\right)$.

As shown in Figure 3, nine studies [5, 6, 10, 14, 17, 21, 22] reported the correction effect by $\mathrm{PO}$, and one [14] of the included studies reported two PO cohorts. Thus, totally ten PO cohorts were pooled into the meta-analysis. Pooled correction degrees in PO cohorts were 31.16 (95\% CI: 26.97-35.35; $\left.I^{2}=81.1 \%, P<0.001\right)$.

As shown in Figure 4, only two studies [10,21] compared the correction effect between AP and PO cohorts, and when 


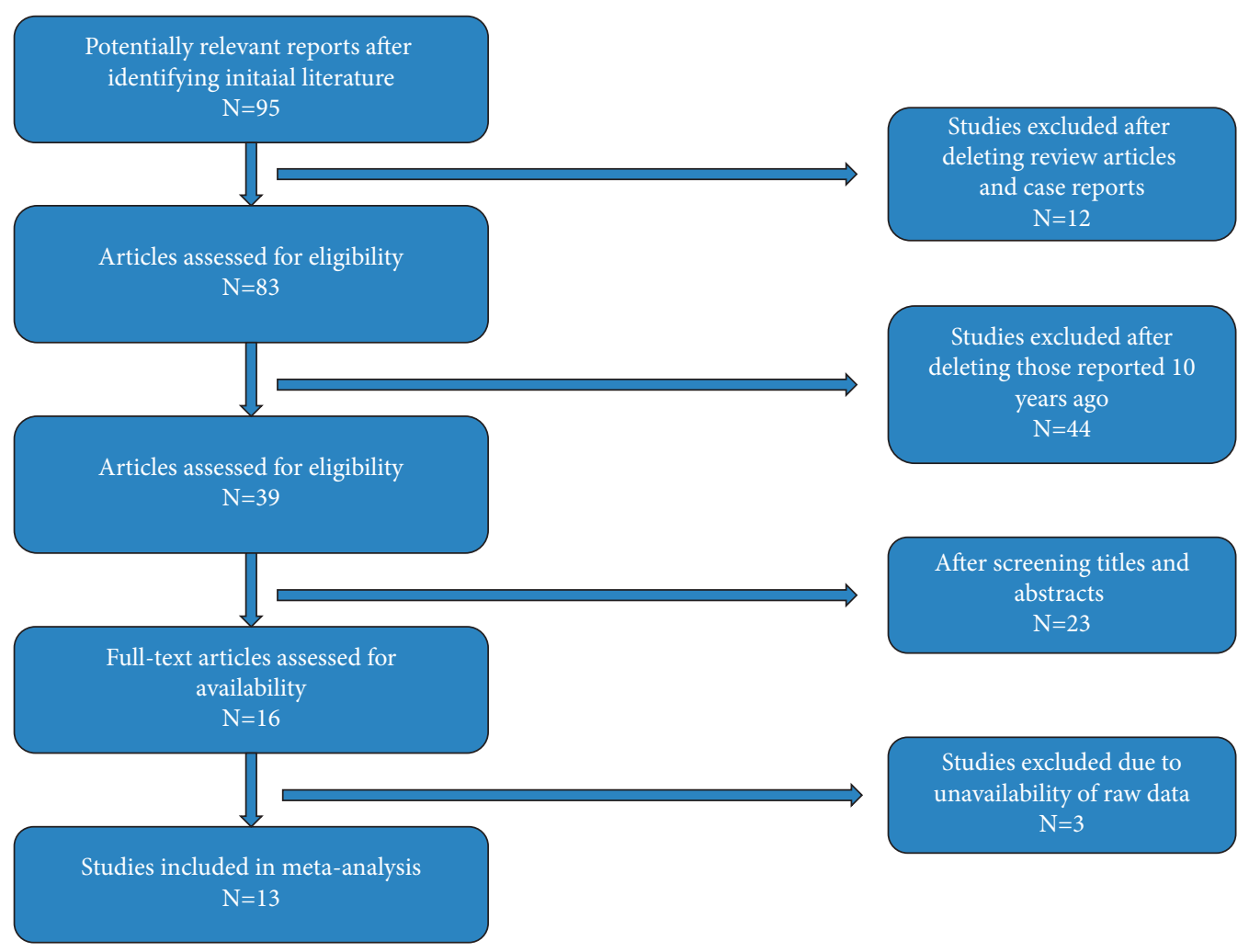

Figure 1: Flow diagram for study selection.

TABLE 1: Quality assessment of included studies by Newcastle-Ottawa Quality Assessment Scale.

\begin{tabular}{lcccc}
\hline Study & Selection & Comparability & Exposure & Total score \\
\hline Dikici et al. [5] & 3 & 2 & 3 & 3 \\
Riouallon et al. [6] & 3 & 2 & 3 & 8 \\
Cobden et al. [4] & 2 & 2 & 3 & 7 \\
Graat et al. [18] & 3 & 2 & 3 & 8 \\
Etemadifar et al. [16] & 4 & 2 & 2 & 9 \\
Faldini et al. [17] & 3 & 2 & 3 & 7 \\
Koller et al. [10] & 3 & 2 & 3 & 8 \\
Koller et al. [19] & 3 & 2 & 3 & 8 \\
Behrbalk et al. [14] & 2 & 2 & 3 & 8 \\
Temponi et al. [21] & 3 & 2 & 3 & 8 \\
Tsutsui et al. [22] & 3 & 2 & 3 & 8 \\
Dasilvaherrero et al. [15] & 3 & 2 & 2 \\
Koptan et al. [20] & 4 & 2 & 3 \\
\hline
\end{tabular}

pooled together for further analysis, the comparison did not indicate any significant difference $(P>0.05)$.

Likewise, only two studies $[16,18]$ compared postoperative PJK incidence between AP and PO cohorts, and pooled analysis of PJK incidence showed no difference, as shown in Figure 5. Four studies [4, 5, 15, 18] have reported incidence of distal junctional kyphosis (DJK), but no studies compared the incidence of postoperative DJK between AP and $\mathrm{PO}$ cohorts. The PJK incidence was reported to range from $0 \%$ to $31 \%$. Also, only two studies $[16,20]$ have reported the surgical data (blood loss and surgical duration), and clearly, the PO approach showed less blood loss and shorter surgical duration as compared to the AP approach.
3.5. Assessment of Pain. As some patients with kyphosis deformity suffer from back pain, we here also incorporated the pain assessment based on the available data. Among all studies included, 4 studies have assessed pain status change and recorded as visual analogue scale (VAS) score $[6,18,20,21]$. Riouallon et al. [6] performed a comparative study including 131 patients, 79\% cases undergoing correction surgeries because of severe back pain and $21 \%$ due to cosmetic disorders. They followed up 85 patients for more than one year after surgeries and found that most patients $(81 \%)$ did not suffer postoperative back pain but $19 \%$ patients still suffered back pain of different degrees. Graat et al. [18] performed a long-term follow-up of 28 
TABLE 2: Characteristics of included studies.

\begin{tabular}{|c|c|c|c|c|c|c|c|c|c|c|}
\hline \multirow[t]{2}{*}{ Study } & \multirow[t]{2}{*}{ Country } & \multirow{2}{*}{ Study design } & \multicolumn{2}{|c|}{$\begin{array}{l}\text { Follow-up } \\
\text { (months) }\end{array}$} & \multicolumn{2}{|c|}{ Age (yrs) } & \multicolumn{2}{|c|}{ Sex } & \multicolumn{2}{|c|}{$\begin{array}{c}\text { No. of } \\
\text { patients }\end{array}$} \\
\hline & & & AP & $\mathrm{PO}$ & $\mathrm{AP}$ & $\mathrm{PO}$ & M & $\mathrm{F}$ & $\mathrm{AP}$ & $\mathrm{PO}$ \\
\hline Dikici et al. [5] & Turkey & Retrospective & - & 36 & - & $18.6 \pm 3.4$ & 20 & 19 & - & 39 \\
\hline Riouallon et al. [6] & France & Retrospective & 57 & 57 & Overall: & $23 \pm 10$ & 81 & 50 & 64 & 67 \\
\hline Cobden et al. [4] & Turkey & Retrospective & - & 41 & - & $19(15-36)$ & 18 & 2 & - & 20 \\
\hline Graat et al. [18] & Netherlands & Cohort study & 216 & 216 & Overall & $44 \pm 8$ & - & - & 16 & 13 \\
\hline Etemadifar et al. [16] & Iran & Prospective & 69.6 & 45.6 & $20.9 \pm 5.3$ & $19.3 \pm 2.7$ & 20 & 10 & 16 & 14 \\
\hline Faldini et al. [17] & Italy & Retrospective & - & 25.2 & - & $19.6(13-24)$ & - & - & - & 20 \\
\hline Koller et al. [10] & Germany/US & Matched-pair study & - & - & $23.6 \pm 11.4$ & $20.7 \pm 10.4$ & - & - & 46 & 46 \\
\hline Koller et al. [19] & Germany & Retrospective & 24 & - & $23.6 \pm 10.8$ & - & 74 & 37 & 111 & - \\
\hline Behrbalk et al. [14] & UK & Retrospective & - & $\geq 24$ & - & $22 \pm 8$ & 8 & 2 & - & 10 \\
\hline Behrbalk et al. [14] & UK & Retrospective & - & $\geq 24$ & - & $19 \pm 6$ & 10 & 1 & - & 11 \\
\hline Temponi et al. [21] & Brazil & Case-control & 37.5 & 22.8 & 19 & 27.3 & 22 & 6 & 19 & 9 \\
\hline Tsutsui et al. [22] & US & Retrospective & - & - & $15.1(13-17)$ & $14.8(11-19)$ & 13 & 9 & 11 & 11 \\
\hline Dasilvaherrero et al. [15] & Brazil & Retrospective & - & $65.8 \pm 39.92$ & - & $16.8 \pm 2.89$ & 7 & 3 & - & 10 \\
\hline Koptan et al. [20] & Egypt & Retrospective & $\geq 24$ & $\geq 24$ & $16 \pm 0.7$ & $15 \pm 0.6$ & 12 & 21 & 17 & 16 \\
\hline
\end{tabular}

$\mathrm{AP}$, combined anterior-posterior approach; PO, posterior-only approach; OP, operation; M, male; F, female.

TABLE 3: Kyphosis correction of the patients included in all studies.

\begin{tabular}{|c|c|c|c|c|c|c|c|c|}
\hline \multirow{2}{*}{ Study } & \multicolumn{2}{|c|}{ Cobb angle (pre-op) } & \multicolumn{2}{|c|}{ Cobb angle (post-op) } & \multicolumn{2}{|c|}{ Correction (degree) } & \multicolumn{2}{|c|}{ Correction rate } \\
\hline & $\mathrm{AP}$ & $\mathrm{PO}$ & $\mathrm{AP}$ & $\mathrm{PO}$ & $\mathrm{AP}$ & $\mathrm{PO}$ & $\mathrm{AP}$ & $\mathrm{PO}$ \\
\hline Dikici et al. [5] & - & $73.3 \pm 7.9$ & - & $39 \pm 8.7$ & - & - & - & $46 \% \pm 13$ \\
\hline Riouallon et al. [6] & $76 \pm 23$ & $78 \pm 13$ & $57 \pm 21$ & $61 \pm 14$ & - & - & - & - \\
\hline Cobden et al. [4] & - & 79.8 & - & 44.9 & - & - & - & - \\
\hline Graat et al. [18] & 85 & 79 & 62.1 & 65.6 & - & - & $27 \%$ & $17 \%$ \\
\hline Etemadifar et al. [16] & $83.7 \pm 8.1$ & $81.9 \pm 9.4$ & $43 \pm 7.5$ & $43.2 \pm 9.8$ & 42.2 & 41.8 & $50.5 \%$ & $51 \%$ \\
\hline Faldini et al. [17] & - & $78.6 \pm 11.2$ & - & $45.8 \pm 4.4$ & - & - & - & - \\
\hline Koller et al. [10] & $75.9 \pm 9.6$ & $78.7 \pm 10.1$ & $43.4 \pm 12.3$ & $47.1 \pm 11.7$ & $33.7 \pm 14.7$ & $30.6 \pm 12.4$ & - & - \\
\hline Koller et al. [19] & $67.2 \pm 12.2$ & - & $38.5 \pm 14.8$ & - & $28.9 \pm 13.4$ & - & - & - \\
\hline Behrbalk et al. [14] [1] & - & $72 \pm 7$ & - & $43 \pm 9$ & - & $29 \pm 9$ & - & - \\
\hline Behrbalk et al. [14] [2] & - & $78 \pm 9$ & - & $44 \pm 8$ & - & $34 \pm 6$ & - & - \\
\hline Temponi et al. [21] & $77.6 \pm 10.4$ & $72.9 \pm 12.0$ & $35.8 \pm 8.0$ & $44.3 \pm 9.8$ & $41.7 \pm 12$ & $28.6 \pm 6$ & $53.2 \pm 11.9$ & $39.3 \pm 7.8$ \\
\hline Tsutsui et al. [22] & $84.9 \pm 10.2$ & $82.7 \pm 6.4$ & $48.6 \pm 5.7$ & $47.9 \pm 5.4$ & - & - & - & - \\
\hline $\begin{array}{l}\text { Dasilvaherrero et al. } \\
\text { [15] }\end{array}$ & - & $78.8 \pm 7.59$ & - & $47.5 \pm 12.54$ & - & $33.9 \pm 9.53$ & - & $43.25 \% \pm 12.56 \%$ \\
\hline Koptan et al. [20] & $79.8(65-98)$ & $\begin{array}{c}85.5 \\
(69-102)\end{array}$ & - & - & $38.8(37-45)$ & $45.1(40-49)$ & $48.7 \%$ & $52.2 \%$ \\
\hline
\end{tabular}

AP, combined anterior-posterior approach; PO, posterior-only approach; op, operation.

TABLE 4: Other information of the patients in all included studies.

\begin{tabular}{|c|c|c|c|c|c|c|c|c|}
\hline \multirow{2}{*}{ Study } & \multicolumn{2}{|c|}{ Blood loss (mL) } & \multicolumn{2}{|c|}{ Surgical duration } & \multicolumn{2}{|c|}{ PJK (case) } & \multicolumn{2}{|c|}{ DJK (case) } \\
\hline & $\mathrm{AP}$ & $\mathrm{PO}$ & AP & $\mathrm{PO}$ & $\mathrm{AP}$ & $\mathrm{PO}$ & AP & $\mathrm{PO}$ \\
\hline Dikici et al. [5] & - & - & - & - & - & - & - & $12(31 \%)$ \\
\hline Riouallon et al. [6] & - & - & - & - & - & - & - & - \\
\hline Cobden et al. [4] & - & - & - & - & - & $3(15 \%)$ & - & $3(15 \%)$ \\
\hline Graat et al. [18] & - & - & - & - & 9 & 6 & 0 & 0 \\
\hline Etemadifar et al. [16] & 1380 & 760 & $545.3 \mathrm{~min}$ & $263.5 \mathrm{~min}$ & 1 & 1 & - & - \\
\hline Faldini et al. [17] & - & - & - & - & - & - & - & - \\
\hline Koller et al. [10] & - & - & - & - & - & - & - & - \\
\hline Koller et al. [19] & - & - & - & - & - & - & - & - \\
\hline Behrbalk et al. [14] & - & - & - & - & - & - & - & - \\
\hline Temponi et al. [21] & - & - & - & - & - & - & - & - \\
\hline Tsutsui et al. [22] & - & - & - & - & - & - & - & - \\
\hline Dasilvaherrero et al. [15] & - & - & - & - & - & 1 & - & 0 \\
\hline Koptan et al. [20] & 910 & 620 & $315 \mathrm{~min}$ & $215 \mathrm{~min}$ & - & - & - & - \\
\hline
\end{tabular}

AP, combined anterior-posterior approach; PO, posterior-only approach; op, operation; PJK, proximal junctional kyphosis; DJK, distal junctional kyphosis. 


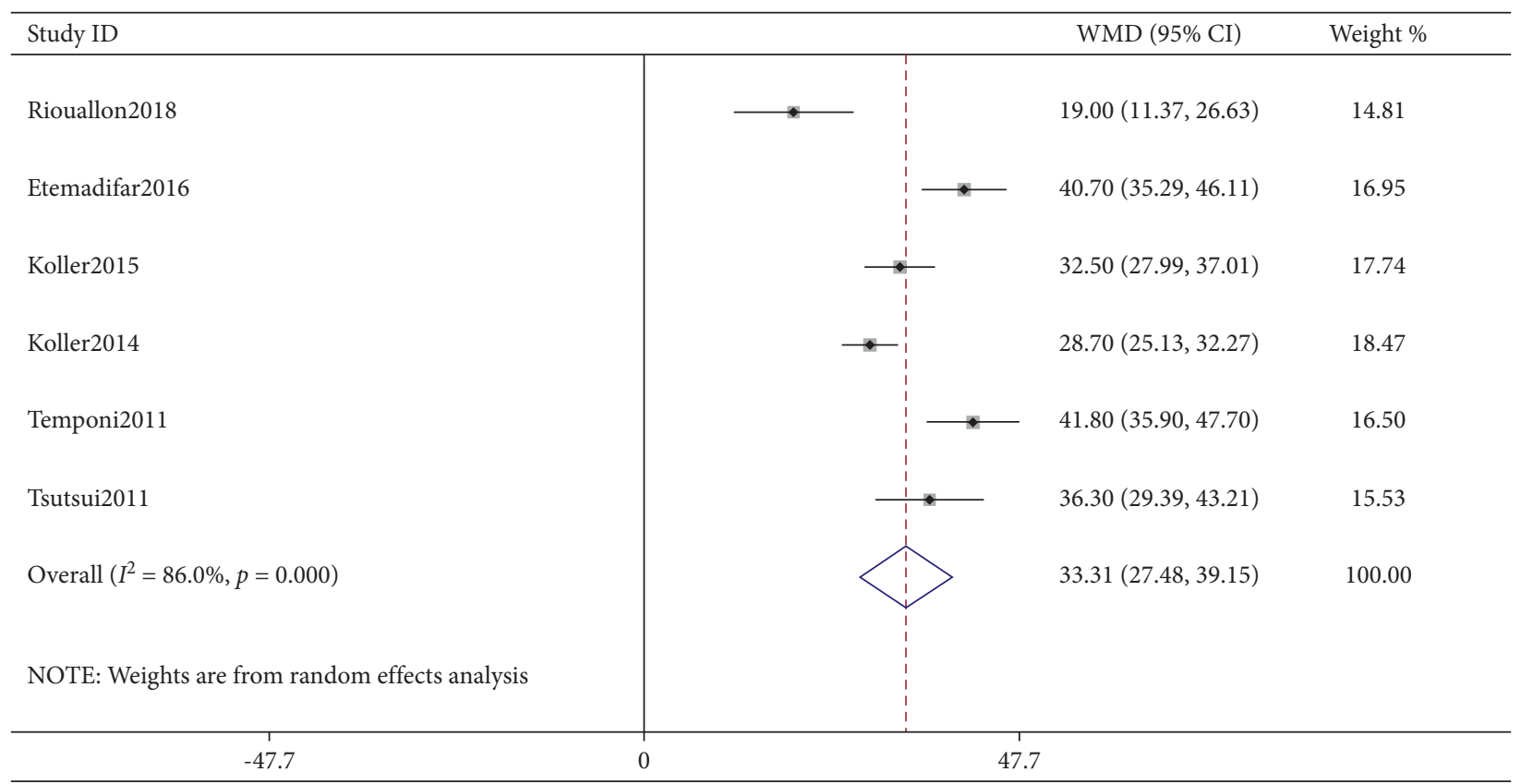

FIGURE 2: Forest plot of kyphosis correction by the combined anterior-posterior approach.

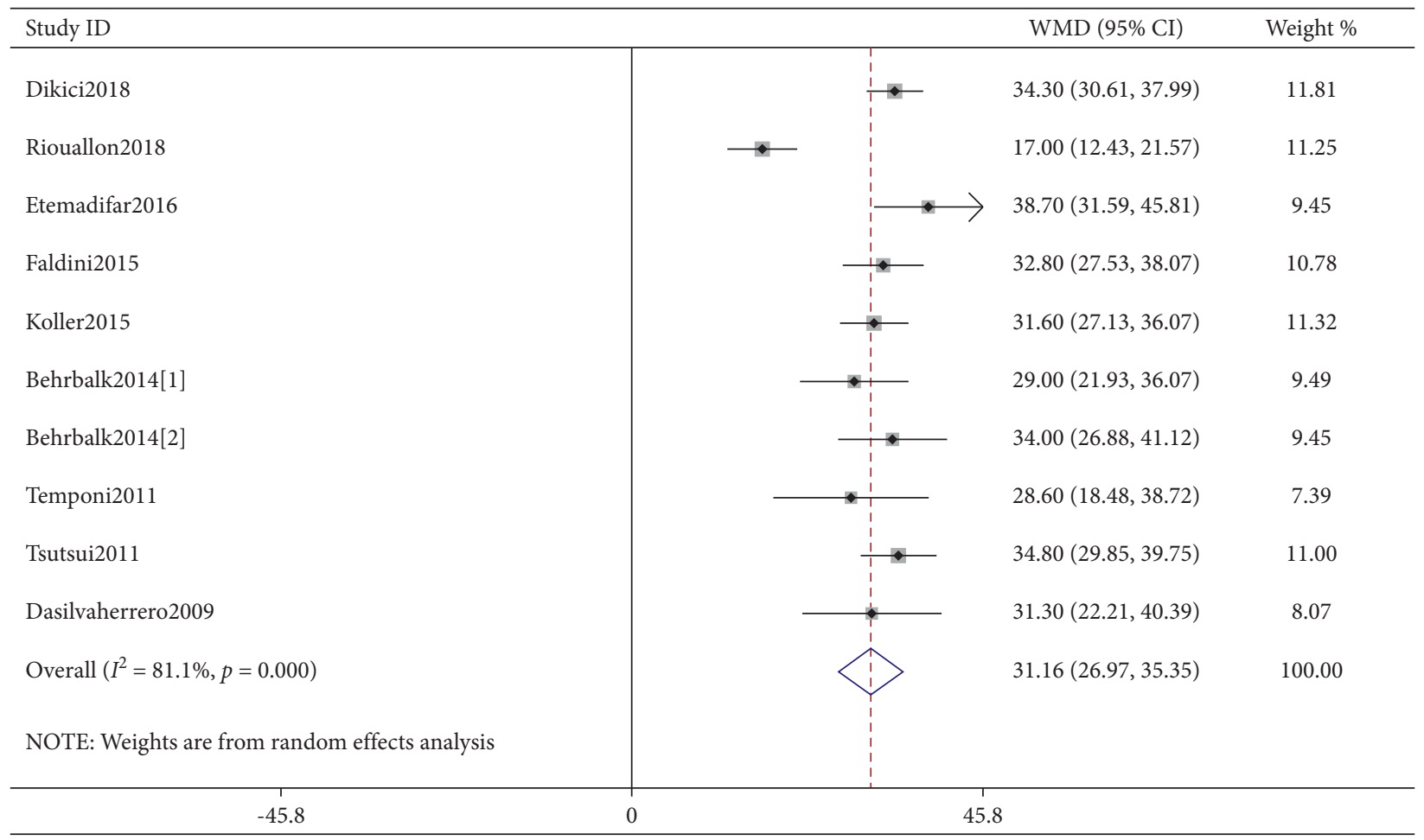

FIGURE 3: Forest plot of kyphosis correction by the posterior-only approach.

patients postsurgery and compared them regarding back pain; it was found that the AP group suffered less pain than the PO group, while Temponi et al. [21] reported the opposite result to that. Koptan et al. [20] reported that all patients complained of pain preoperatively but did not give further information.
3.6. Publication Bias Assessment. As shown in Figure 6, no publication bias was found relevant to correction of kyphosis in AP cohorts by Begg's rank correlation test and Egger's linear regression test (both $P>0.10$ ). Likewise, Figure 7 showed no publication bias with regard to correction of kyphosis in PO cohorts (both $P>0.10$ ). 


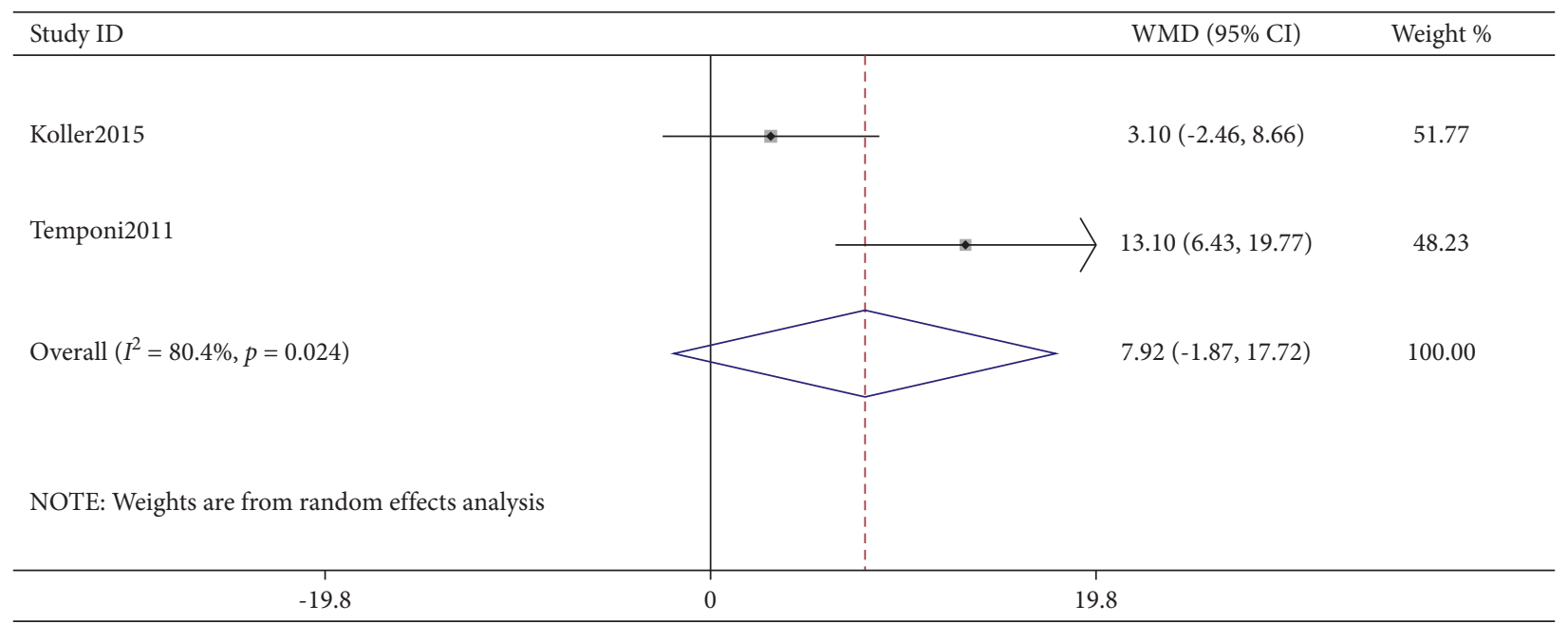

Figure 4: Comparison of kyphosis correction between the combined anterior-posterior approach and the posterior-only approach.

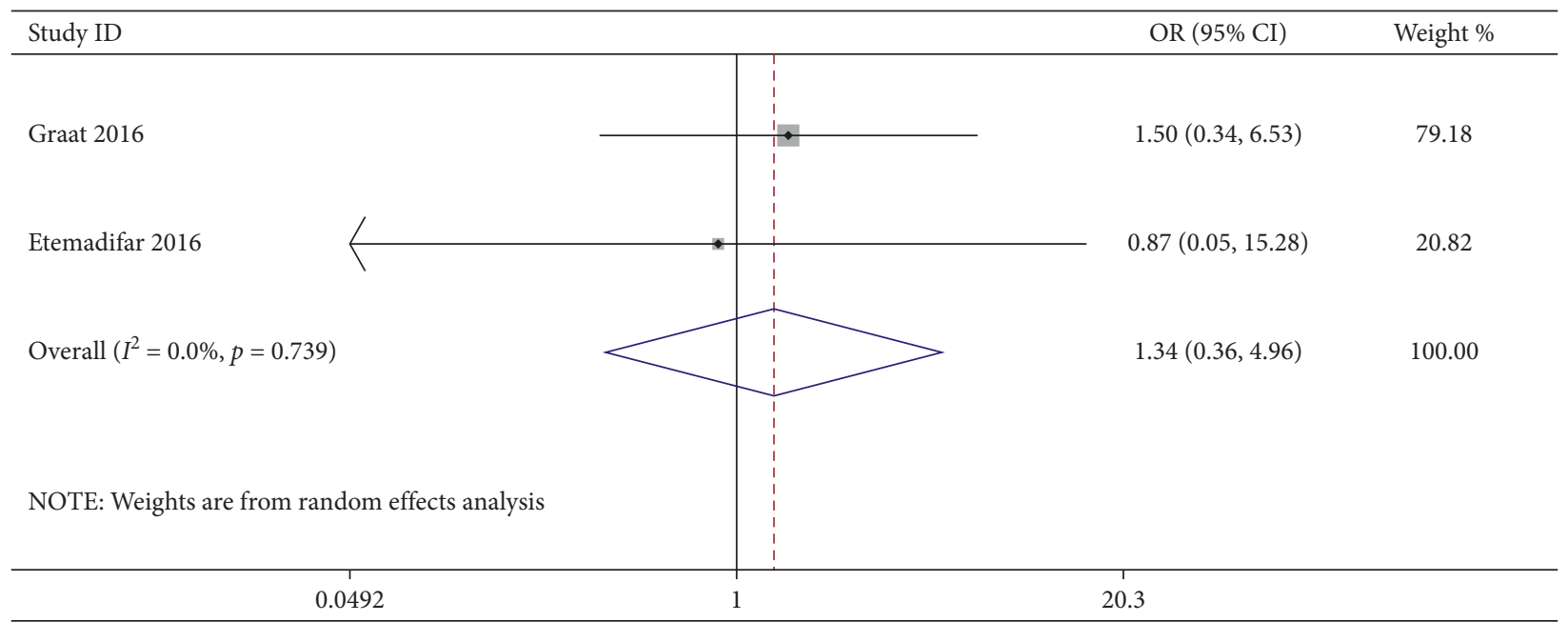

FIGURE 5: Comparison of proximal junctional kyphosis (PJK) incidence between the combined anterior-posterior approach and the posterior-only approach.

\section{Discussion}

To the best of our knowledge, the PO approach was the first surgical technique introduced to correct SK deformity and was first performed by Bradford in 1975 [4, 26]. Many clinical and radiological results have reported that PO fusion is an efficient technique for the treatment of SK $[4,6,10,14,16,17,21]$. Different methods have been introduced over the past few years, and combined AP fusion has been recommended more suitable for rigid and major deformities for many years $[10,19]$, but complication rates, operation time, and blood loss were significantly higher in AP procedures [16]. Nowadays, debates continue regarding surgical strategy selection between AP and PO fusion for the surgical management of SK [27].

In this study, we performed a meta-analysis in an effort to identify a better approach from AP and PO fusion procedures for correcting SK deformity. The research focus was on the correction effect reflected by achieving more correction degrees, and postoperative complications including PJK were also compared between the two groups, although DJK cannot be compared due to the lack of reports. In our study, six studies reported the correction effect by AP and were pooled into the meta-analysis. As a result, pooled correction effect in AP cohorts was 33.31 degrees (WMD, 95\% CI: 27.48-39.15). In addition, nine studies reported the correction effect by $\mathrm{PO}$, and one of them reported two PO cohorts. Thus, totally ten PO cohorts were pooled into the meta-analysis. Also, pooled correction effect in PO cohorts was 31.16 degrees (WMD, 95\% CI: 26.97-35.35). Comparing the correction effect between the AP approach and the PO approach, there was no significant difference found although only two studies compared AP cohorts to PO cohorts $(P>0.05)$. Likewise, only two studies compared postoperative PJK incidence between AP and PO cohorts, and pooled analysis of PJK incidence showed no difference. 

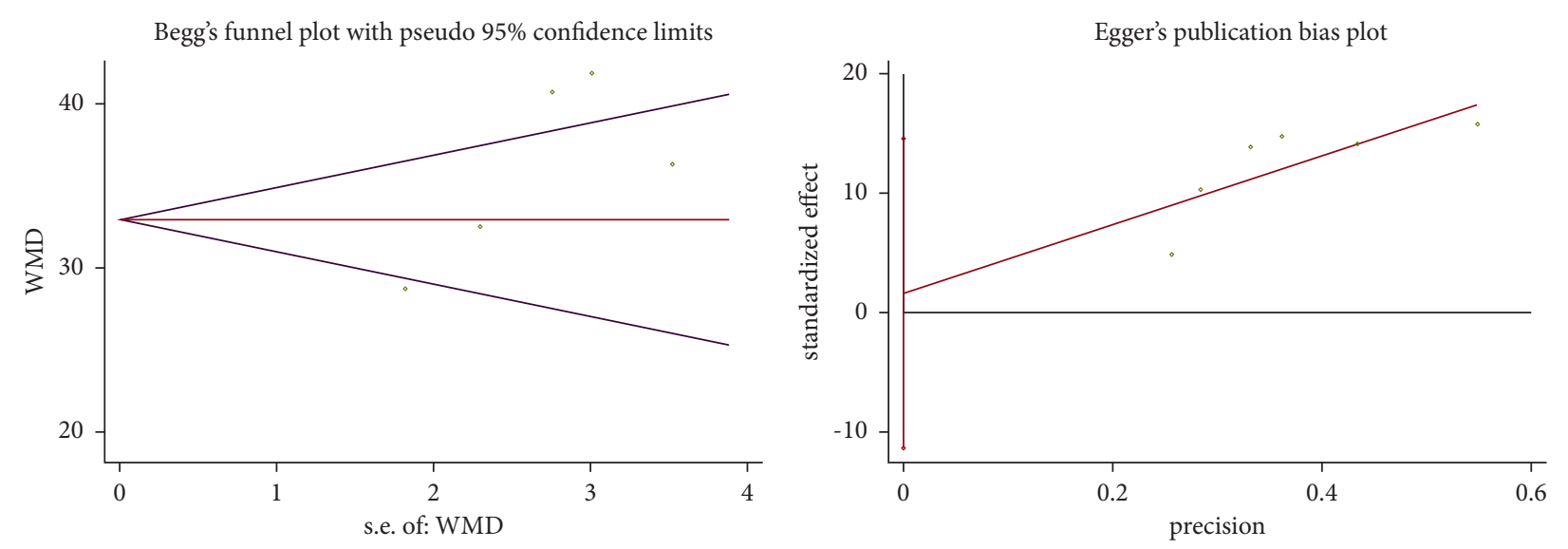

$$
\begin{aligned}
& \text { Tests for Publication Bias } \\
& \text { Begg's Test } \\
& \text { adj. Kendall's Score (P-Q) }=r \begin{aligned}
& \\
& \text { Std. Dev. of score }= 5.32 \\
& \text { Number of Studies }=6 \\
& \mathrm{z}=0.19 \\
& \operatorname{Pr}>|\mathrm{z}|=0.851 \\
& \mathrm{z}=0.00 \quad \text { (continuity corrected) } \\
& \operatorname{Pr}>|\mathrm{z}|=1.000 \quad \text { (continuity corrected) }
\end{aligned}
\end{aligned}
$$

\begin{tabular}{l|cccccc}
\multicolumn{1}{l}{ Egger's test } & \multicolumn{1}{l}{} \\
\hline Std_Eff & Coef. & Std. Err. & $\mathrm{t}$ & $\mathrm{p}>|\mathrm{t}|$ & \multicolumn{2}{c}{ [95\% Conf. Interval] } \\
\hline slope & 28.81027 & 12.22735 & 2.36 & 0.078 & -5.138296 & 62.75883 \\
bias & 1.628813 & 4.679757 & 0.35 & 0.745 & -11.36428 & 14.6219 \\
\hline
\end{tabular}

Figure 6: Publication bias analysis by Begg's and Egger's tests regarding the combined anterior-posterior approach.

Unfortunately, there were no studies having compared the incidence of postoperative DJK between AP and PO cohorts, though four studies have demonstrated DJK incidence ranging from $0 \%$ to $31 \%$. Also, only two studies have reported the surgical data (blood loss and surgical duration), and apparently, the PO approach showed less blood loss and shorter surgical duration.

A previous meta-analysis [7] has revealed that the pooled correction loss of Cobb angle for the AP group was 4.1 (95\% CI: 3.4-4.8), and for the PO group, it was 3.8 (95\% CI: 3.3-4.4), without any significant difference indicated by the results. This report is consistent with our meta-analysis results that there was no difference with regard to the change of Cobb angle before and after surgery between the AP group and the PO group. Moreover, it was reported that the PO group showed advantages in blood loss, surgery time, and junctional kyphosis [7]. It was in line with our results that the PO group showed less blood loss and shorter surgery duration. Our analytical results, however, did not indicate any difference regarding the postoperative PJK incidence due to the lack of raw data that were available. That metaanalysis has included a wide range of studies that were published between 1964 and 2012, and those studies varied too much, especially considering that the surgical techniques are ongoing in progress. To overcome the shortcomings, we only included eligible studies published between 2009 and
2020 , within around recent ten years. Seven new published articles $[4,6,10,16-18]$ have been included in our metaanalysis, which is a helpful update to that previous metaanalysis [7]. Recently, another meta-analysis showed that PO surgery and AP surgery achieved comparable treatment effects of SK disease, which is consistent with our results [28]. However, that study goes with the limitations that most of the studies included were published ten years ago, and thus that meta-analysis missed some important up-to-date literature.

As to publication bias assessment in this study, there were no publication bias found relating to correction of kyphosis in AP cohorts by Begg's rank correlation test and Egger's linear regression test (both $P>0.10$ ). Likewise, it also showed no publication bias with regard to the correction of kyphosis in PO cohorts (both $P>0.10$ ). Thus, this metaanalysis is in a good quality in terms of publication bias.

However, we have to demonstrate some potential limitations that may exist in this work. To start with, only English-written studies were selected and included in this meta-analysis, potentially excluding some relevant reports written in other languages, due to a language limitation. Additionally, the number of patients included in both groups was relatively small (AP: 300 vs. PO: 286), which cannot be neglected in the interpretation of findings in this meta-analysis. At last, all included studies in the pooled 

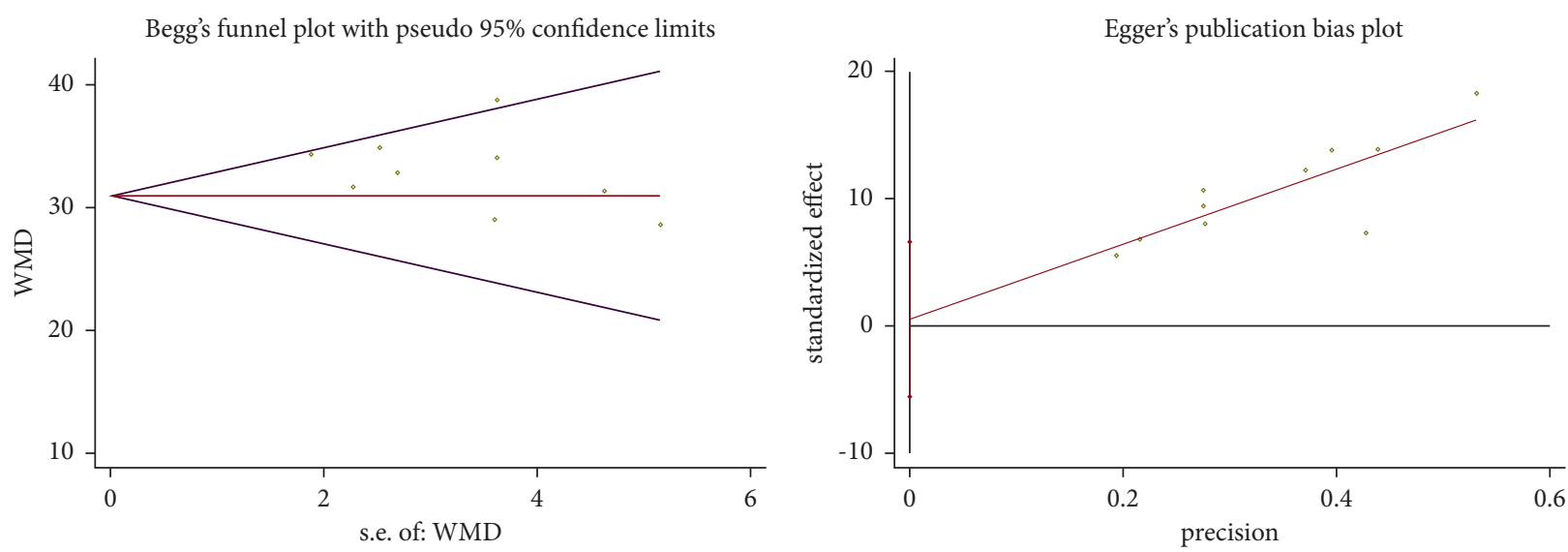

Tests for Publication Bias

Begg's Test

adj. Kendall's Score $(\mathrm{P}-\mathrm{Q})=-7$

Std. Dev. of Score $=11.18$

Number of Studies $=\quad 10$

$z=-0.63$

$\operatorname{Pr}>|z|=0.531$

$\mathrm{z}=0.54$ (continuity corrected)

$\operatorname{Pr}>|z|=0.592 \quad$ (continuity corrected)

\begin{tabular}{l|cccccc}
\multicolumn{1}{l}{ Egger's test } & \multicolumn{1}{c}{} \\
\hline Std_Eff & Coef. & Std. Err. & $\mathrm{t}$ & $\mathrm{p}>|\mathrm{t}|$ & \multicolumn{2}{c}{ [95\% Conf. Interval] } \\
\hline slope & 29.52363 & 7.41756 & 3.98 & 0.004 & 12.4187 & 46.62855 \\
bias & .5211755 & 2.639498 & 0.20 & 0.848 & -5.565518 & 6.607869 \\
\hline
\end{tabular}

Figure 7: Publication bias analysis by Begg's and Egger's tests regarding the posterior-only approach.

analysis were retrospective in design and most are noncomparative studies, thus might reduce the power of this work.

\section{Conclusions}

In summary, this meta-analysis shows similar treatment effects between AP and PO procedures in correcting Scheuermann's kyphosis, suggesting the advantage of PO procedures due to less blood loss and surgical duration. However, the postoperative complications PJK and DJK cannot be well concluded due to the limitation of existing reports.

\section{Abbreviations}

AP: Anterior-posterior

CI: Confidence interval

DJK: Distal junctional kyphosis

OR: Odds ratio

PJK: Proximal junctional kyphosis

PO: Posterior-only

SK: Scheuermann's kyphosis

WMD: Weighted mean difference.

\section{Data Availability}

The data used in this study are all included within this article and open to all readers.

\section{Conflicts of Interest}

All authors declare no conflicts of interest regarding this study.

\section{References}

[1] S. Wang, L. Xu, and M. Wang, "Contribution of postoperative vertebral remodeling to reversal of vertebral wedging and prevention of correction loss in patients with adolescent Scheuermann's kyphosis," Journal of Neurosurgery. Spine, vol. 2021, pp. 1-9, 2021.

[2] L. Vital, B. Nunes, S. A. Santos, V. Veludo, F. Serdoura, and A. Pinho, "Sagittal plane alignment and functional outcomes following surgery for scheuermann kyphosis," Revista brasileira de ortopedia, vol. 56, no. 4, pp. 446-452, 2021.

[3] V. Arlet and D. Schlenzka, "Scheuermann's kyphosis: surgical management," European Spine Journal: Official Publication of the European Spine Society, vol. 14, no. 9, pp. 817-827, 2005.

[4] A. Cobden, A. Albayrak, Y. Camurcu, H. Sofu, T. Tacal, and M. A. Kaygusuz, "Posterior-only approach with pedicle screws for the correction of Scheuermann's kyphosis," Asian spine journal, vol. 11, no. 4, pp. 513-519, 2017.

[5] F. Dikici, T. Akgul, and K. Sariyilmaz, "Selection of distal fusion level in terms of distal junctional kyphosis in Scheuermann kyphosis. A comparison of 3 methods," Acta Orthopaedica et Traumatologica Turcica, vol. 52, no. 1, pp. 7-11, 2018. 
[6] G. Riouallon, C. Morin, and Y. P. Charles, "Posterior-only versus combined anterior/posterior fusion in Scheuermann disease: a large retrospective study," European Spine Journal, vol. 27, no. 9, pp. 2322-2330, 2018.

[7] C. Yun and C. L. Shen, "Anterior release for Scheuermann's disease: a systematic literature review and meta-analysis," European Spine Journal, vol. 26, no. 3, pp. 921-927, 2017.

[8] D. S. Bradford, K. B. Ahmed, J. H. Moe, R. B. Winter, and J. E. Lonstein, "The surgical management of patients with Scheuermann's disease: a review of twenty-four cases managed by combined anterior and posterior spine fusion," The Journal of Bone and Joint Surgery, vol. 62, no. 5, pp. 705-712, 1980.

[9] S. S. Lee, L. G. Lenke, and T. R. Kuklo, "Comparison of Scheuermann kyphosis correction by posterior-only thoracic pedicle screw fixation versus combined anterior/posterior fusion," Spine, vol. 31, no. 20, pp. 2316-2321, 2006.

[10] H. Koller, L. G. Lenke, and O. Meier, "Comparison of anteroposterior to posterior-only correction of Scheuermann's kyphosis: a matched-pair radiographic analysis of 92 patients," Spine deformity, vol. 3, no. 2, pp. 192-198, 2015.

[11] F. Y. Liu, S. D. Yang, L. S. Huo, T. Wang, D. L. Yang, and W. Y. Ding, "Laminoplasty versus laminectomy and fusion for multilevel cervical compressive myelopathy: a meta-analysis," Medicine, vol. 95, no. 23, p. e3588, 2016.

[12] J. Yang, X. Hu, Q. Zhang, H. Cao, J. Wang, and B. Liu, "Homocysteine level and risk of fracture: a meta-analysis and systematic review," Bone, vol. 51, no. 3, pp. 376-382, 2012.

[13] S. D. Yang, Q. Chen, and H. K. Wei, "Bone fracture and the interaction between bisphosphonates and proton pump inhibitors: a meta-analysis," International Journal of Clinical and Experimental Medicine, vol. 8, no. 4, pp. 4899-4910, 2015.

[14] E. Behrbalk, O. Uri, R. M. Parks, M. P. Grevitt, M. Rickert, and B. M. Boszczyk, "Posterior-only correction of Scheuermann kyphosis using pedicle screws: economical optimization through screw density reduction," European Spine Journal, vol. 23, no. 10, pp. 2203-2210, 2014.

[15] C. F. da Silva Herrero, M. A. Porto, M. H. Barbosa, and H. L. Defino, "Multiple segmental osteotomies to the KYPHOSIS correction," Revista Brasileira de Ortopedia, vol. 44, no. 6, pp. 513-518, 2009.

[16] M. Etemadifar, A. Ebrahimzadeh, A. Hadi, and M. Feizi, "Comparison of Scheuermann's kyphosis correction by combined anterior-posterior fusion versus posterior-only procedure," European Spine Journal, vol. 25, no. 8, pp. 2580-2586, 2016.

[17] C. Faldini, F. Traina, F. Perna, R. Borghi, K. Martikos, and T. Greggi, "Does surgery for Scheuermann kyphosis influence sagittal spinopelvic parameters," European Spine Journal, vol. 24, pp. 893-897, 2015.

[18] H. C. Graat, J. J. Schimmel, R. J. Hoogendoorn, L. van Hessem, A. Hosman, and M. de Kleuver, "Poor radiological and good functional long-term outcome of surgically treated scheuermann patients," Spine, vol. 41, no. 14, pp. E869-E878, 2016.

[19] H. Koller, Z. Juliane, M. Umstaetter, O. Meier, R. Schmidt, and W. Hitzl, “Surgical treatment of Scheuermann's kyphosis using a combined antero-posterior strategy and pedicle screw constructs: efficacy, radiographic and clinical outcomes in 111 cases," European Spine Journal, vol. 23, no. 1, pp. 180-191, 2014.

[20] W. M. Koptan, Y. H. Elmiligui, and H. B. Elsebaie, “All pedicle screw instrumentation for Scheuermann's kyphosis correction: is it worth it," The Spine Journal: Official Journal of the North American Spine Society, vol. 9, no. 4, pp. 296-302, 2009.

[21] E. F. Temponi, R. D. de Macedo, L. O. Pedrosa, and B. P. Fontes, "SCHEUERMAN'S KYPHOSIS: comparison between the posterior approach associated with smithpetersen osteotomy and combined anterior-posterior FUSION," Revista brasileira de ortopedia, vol. 46, no. 6, pp. 709-717, 2011.

[22] S. Tsutsui, J. B. Pawelek, T. P. Bastrom, S. A. Shah, and P. O. Newton, "Do discs "open" anteriorly with posterior-only correction of Scheuermann's kyphosis," Spine, vol. 36, no. 16, pp. E1086-E1092, 2011.

[23] A. Ashraf, A. Noelle Larson, D. W. Polly, G. Ferski, K. J. Guidera, and C. H. Mielke, "Change in sagittal plane alignment following surgery for Scheuermann's kyphosis," Spine deformity, vol. 2, no. 5, pp. 404-409, 2014.

[24] J. D. Coe, J. S. Smith, and S. Berven, "Complications of spinal fusion for scheuermann kyphosis: a report of the scoliosis research society morbidity and mortality committee," Spine, vol. 35, no. 1, pp. 99-103, 2010.

[25] A. Jain, P. D. Sponseller, K. M. Kebaish, and A. Mesfin, "National trends in spinal fusion surgery for scheuermann kyphosis," Spine deformity, vol. 3, no. 1, pp. 52-56, 2015.

[26] C. Palazzo, F. Sailhan, and M. Revel, "Scheuermann's disease: an update," Joint Bone Spine: revue du rhumatisme, vol. 81, no. 3, pp. 209-214, 2014.

[27] A. I. Tsirikos and A. K. Jain, "Scheuermann's kyphosis; current controversies," Journal of Bone and Joint Surgery British Volume, vol. 93, no. 7, pp. 857-864, 2011.

[28] C. H. Lee, Y. I. Won, and Y. San Ko, "Posterior-only versus combined anterior-posterior fusion in Scheuermann disease: a systematic review and meta-analysis," Journal of Neurosurgery, Spine, vol. 25, pp. 1-9, 2020. 